\title{
Germanica
}

\section{Retour sur une influence supposée}

Leutnant Gustl d'Arthur Schnitzler et Les Lauriers sont coupés d'Édouard Dujardin

Reflections about a presumed influence : Leutnant Gustl et Les lauriers sont coupés

Überlegungen zu einem mutmaßlichen Einfluss : Leutnant Gustl und Les Lauriers sont coupés

\section{Frédéric Teinturier}

\section{(2) OpenEdition}

\section{Journals}

Édition électronique

URL : http://journals.openedition.org/germanica/2125

DOI : 10.4000/germanica.2125

ISSN : 2107-0784

\section{Éditeur}

Université de Lille

\section{Édition imprimée}

Date de publication : 15 juin 2013

Pagination : 101-112

ISBN : 9782913857315

ISSN : 0984-2632

\section{Référence électronique}

Frédéric Teinturier, « Retour sur une influence supposée », Germanica [En ligne], 52 | 2013, mis en ligne le 10 juin 2013, consulté le 06 octobre 2020. URL : http://journals.openedition.org/germanica/2125 ; DOI : https://doi.org/10.4000/germanica.2125 


\title{
Retour sur une influence supposée Leutnant Gustl d'Arthur Schnitzler et Les Lauriers sont coupés d'Édouard Dujardin
}

\author{
Frédéric TEINTURIER \\ Université de Lorraine, Metz.
}

Les récits Leutnant Gustl, d'Arthur Schnitzler et Les Lauriers sont coupés, d'Édouard Dujardin ont été maintes fois étudiés, et sont considérés le plus souvent, à juste titre, comme des œuvres fondatrices, et représentatives d'une certaine modernité narrative. Fait exceptionnel, quasiment unique dans l'histoire de la littérature européenne moderne, ces deux textes narratifs semblent aller de pair, car ils ne sont quasiment jamais évoqués l'un sans l'autre. C'est sur ce phénomène que nous souhaitons revenir plus en détail.

Les études comparées de Leutnant Gustl et de Les Lauriers sont coupés diffèrent parfois fortement mais toutes ont pour objectif de mettre en évidence une ressemblance plus ou moins grande entre les deux œuvres, et toutes partent du constat que le récit de Schnitzler est plus ou moins largement inspiré de celui de Dujardin, dans un mouvement qui pourrait être considéré comme un dialogue culturel. Les ressemblances entre les deux textes sont évidentes et la première d'entre elles a suffi à assurer le succès de la comparaison et à éclipser parfois toute autre interrogation sur la parenté entre les deux récits : il s'agit, on le sait, de l'utilisation systématique par les deux auteurs de ce qu'il est convenu depuis longtemps de nommer la « technique du 
monologue intérieur ». En effet, Leutnant Gustl et Les Lauriers sont coupés se présentent comme deux récits à la première personne, récits dont l'objet exclusif est la transcription des pensées et des paroles d'un seul personnage. Aucune autre perspective narrative n'est présente dans les deux cas, ni aucune entorse n'est constatée dans l'application de cette technique.

Au-delà de cette utilisation commune et moderne du récit monologique exclusivement consacré à la mise en scène des pensées du seul narrateur, la comparaison entre les deux œuvres s'est imposée parce que Schnitzler n'a pas caché son intérêt - relatif, d'ailleurs - pour l'expérience narrative tentée par son aîné. Rappelons les faits, qui sont connus : Schnitzler évoque au moins deux fois dans sa correspondance l'inspiration qu'il tient de Dujardin ; une première fois dans une lettre à Maria Reinhard, datée de 1898 ; une seconde fois dans une lettre à Georg Brandes souvent citée, et datée de 1901. À son amie, Schnitzler parle du caractère unique et exceptionnel du livre de Dujardin, qu'il vient de lire ${ }^{1}$, au critique danois qui lui témoigne son intérêt pour Leutnant Gustl, il avoue sans détour :

Ich freue mich, dass Sie die Novelle vom Leutnant Gustl amüsiert hat. Eine Novelle von Dostojewski, Krotkaja, die ich nicht kenne, soll die gleiche Technik des Gedankenmonologs aufweisen. Mir aber wurde der erste Anlass zu der Form durch eine Geschichte von Dujardin gegeben, betitelt Les lauriers sont coupés. Nur dass dieser Autor für seine Form nicht den rechten Stoff zu finden wusste ${ }^{2}$.

C'est donc avant tout en raison de la forme choisie que Schnitzler s'intéresse à l'œuvre de Dujardin, qu'il trouve peu convaincante par ailleurs. Malgré cette précieuse indication, les études sont peu nombreuses qui analysent de manière systématique l'usage qui est fait par ces deux auteurs de la technique du monologue intérieur ${ }^{3}$ : cette parenté serait somme toute évidente, comme le sont, de manière tout aussi évidente, les différences entre les deux textes. Pourtant, l'évidence revendiquée par Schnitzler du lien qui relie son texte à celui de Dujardin ne doit pas nous faire renoncer à interroger la nature même de cette parenté, son origine tout autant que ses limites, qui sont

1. - « eine sehr eigenartige Geschichte », Arthur Schnitzler, Briefe 1875-1912, hg. V. Therese Nickl und Heinrich Schnitzler, Frankfurt a.M., Fischer, 1981, p. 374.

2. - Georg Brandes und Arthur Schnitzler, Ein Briefwechsel, hg. von Kurt Bergel, Bern, Francke, 1956, p. 87-88.

3. - On retiendra ainsi l'étude de Theodor W. et Beatrice Alexander : «Schnitzler's Leutnant Gustl and Dujardin's Les lauriers sont coupés », in : Modern Austrian Literature, 2/2, 1969, p. 7-15 ; citons aussi Barbara Surowska: « Schnitzlers innerer Monolog im Verhältnis zu Dujardin und Dostojewski » in : Theatrum Europaeum. Festschrift für E. M. Szarota, hg. von Richard Brinkmann, Munich, Fink, 1982, p. 549-558. 
tout aussi révélatrices. Notre propos consiste ainsi à évaluer la validité de la comparaison entre les deux textes. La genèse de la nouvelle de Schnitzler, rédigée en 1900, comme en atteste une lettre de l'auteur à Hofmannsthal ${ }^{4}$, focalise d'emblée les regards interprétatifs sur cette comparaison. Mais en quoi le simple fait que Schnitzler ait recours à la technique expérimentée une première fois par Dujardin justifie-t-il de considérer un tel rapprochement entre les deux récits comme pertinent et, ce qui est souvent la conséquence de ce rapprochement, en quoi cela justifie-t-il que l'on parle de dialogue, d'influence ou simplement d'inspiration? A contrario, on pourrait en fait se demander - du moment que l'on se concentre sur l'aspect purement technique de l'apparence narrative des textes - pourquoi personne n'a encore cherché à comparer Les Lauriers sont coupés et une autre nouvelle de Schnitzler, qui utilise elle aussi et de manière plus troublante encore cette même technique du monologue intérieur exclusif : Fräulein Else. La question est sans doute un peu provocante : on comprend cependant aisément ainsi la validité de la comparaison entre Gustl et Les lauriers laquelle, contrairement à celle qui pourrait résulter du rapprochement avec Fräulein Else, repose aussi et surtout sur une ressemblance purement thématique. Fräulein Else a beau présenter des caractéristiques techniques en partie similaires, elle est une nouvelle plus tardive, le monde qu'elle décrit n'est plus le même, il ne s'agit pas, comme dans les deux récits qui nous occupent, du monde d'avant 1914. Or sans doute faut-il bien voir dans cette identité historique et culturelle des mondes décrits la justification première d'une comparaison entre nos deux récits, comparaison qui permet en fait d'établir plusieurs points de convergence, au-delà des aspects formels. C'est la double ressemblance technique et historique qui permet d'espérer du rapprochement ainsi opéré qu'il nous révèle la nature et la portée d'un éventuel dialogue entre deux auteurs. Le contenu purement thématique ne fait que confirmer que nous sommes bien face à deux œuvres réellement apparentées : Leutnant Gustl et Les Lauriers sont coupés sont le récit de quelques heures dans la vie d'un homme jeune, désœuvré, qui fréquente le monde des théâtres de la capitale au tournant du siècle et dont les pensées tournent autour des femmes, de l'art, du temps qui passe et de l'état de la société contemporaine. Le ton blasé qui caractérise les deux textes et le tempérament des deux personnages ne font que renforcer un peu plus le lecteur dans son impression d'être en présence de deux récits de fiction très similaires.

Pourtant, une seconde lecture plus systématique des deux textes fait rapidement apparaître des différences majeures, qui laissent à penser

4. - Arthur Schnitzler, Briefe 1875-1912, hg. V. Therese Nickl und Heinrich Schnitzler, Frankfurt a.M., Fischer, 1981, p. 387. ,[Ich] bin jetzt mit einer ziemlich sonderbaren Novelle beschäftigt, die mir viel Freude macht.“ 
que leur évidente parenté n'est pas aussi simple à déterminer, alors même que leur ressemblance reste indiscutable. Leutnant Gustl et Les Lauriers sont coupés témoignent même d'une divergence essentielle entre deux conceptions de la psychologie et de son traitement dans un texte narratif. Il n'est pas essentiel de faire ici une liste des différences qui existent dans le caractère ou la fonction sociale des deux protagonistes, cela relève à l'évidence de divergences mineures au niveau qui occupe. Signalons d'ailleurs qu'on a sans doute raison, inversement, de voir dans certains détails apparemment insignifiants mais « étonnants 5 », présents dans Leutnant Gustl, une éventuelle reprise directe de motifs déjà présents dans Les lauriers sont coupés : on peut penser ainsi à la description du boudoir de Léa, d'une part, et à la mention de la salle de bain de Steffi, d'autre part, telle qu'elle apparait dans le souvenir de Gustl. Les analogies peuvent abonder dans certains détails, venant confirmer la proximité intertextuelle entre les deux histoires, alors même que certains aspects thématiques peut-être plus saillants sont bien différents. Nous avons bien affaire à la réception par Schnitzler du récit de Dujardin, avec les ressemblances et différences qui sont courantes dans un tel cas.

Deux autres points doivent en revanche être examinés plus en détail, qui sont décisifs dans l'analyse de textes narratifs relativement brefs: le rapport à l'espace et au temps. La façon dont un personnage conçoit son existence et l'inscrit dans ce cadre spatio-temporel a des implications essentielles sur la tenue et l'apparence du récit. Et ce d'autant plus directement que le récit dont il est question se présente sous l'apparence exceptionnelle d'une transcription exclusive des pensées d'une seule conscience. Les deux personnages semblent avoir le même rapport au cadre spatial qui les entoure. Cela est en effet frappant, et on peut y voir une justification supplémentaire du rapprochement entre les deux récits. En fait, l'inscription des deux protagonistes dans l'espace est d'autant plus marquante qu'ils ne peuvent littéralement pas rester en place. Prince et Gustl ne cessent d'arpenter la ville - Paris et Vienne. Le récit s'organise donc très précisément en fonction des stations successives des deux hommes. Dans les deux cas, le lecteur peut également suivre pas à pas le parcours des personnages et a comme l'impression de se trouver devant un plan de la ville. La pérégrination semble cependant bien différente, dès lors qu'on entre dans le détail des parcours respectifs. Dans Les Lauriers sont coupés, le récit s'organise en neuf chapitres, et on s'aperçoit rapidement que le rapport aux lieux scande réellement le découpage narratif, puisque chaque chapitre s'achève par un changement ou une tentative de changement de lieu, le suivant s'ou-

5. - Cf. Achim Aurnhammer, Leutnant Gustl, in : Interpretationen Arthur Schnitzler. Dramen und Erzählungen, Stuttgart, Reclam (UB 17532), 2007, p. 81-83. 
vrant immédiatement sur l'arrivée soit dans la rue, au sortir d'un lieu fermé, soit dans une maison, en quittant la rue. Cependant, les différentes stations semblent sans ordre, certaines étant dues au hasard du passage de Prince sur telle ou telle avenue et le parcours n'obéit finalement qu'à la nécessité d'attendre son rendez-vous, qui sera reporté et changé au début du chapitre IV, lorsque son concierge lui tend un billet de Léa, enjoignant son amant de venir la retrouver chez elle. En d'autres termes, il apparaît aisément que l'errance du protagoniste, si elle n'est pas insignifiante, bien au contraire, ne correspond pas, de sa part, à un cheminement conscient et réfléchi. La fin du chapitre I est caractéristique : c'est par hasard, pour tromper son attente, que Prince entre dans le Café de l'Opéra. C'est également par hasard qu'il passe par Saint Augustin, puisqu'il raccompagne un ami rencontré fortuitement dans la rue. Et son changement de direction, son départ de chez lui pour rejoindre Léa chez elle, puis leur promenade en fiacre dans le quartier de celle-ci sont autant d'étapes dont la succession n'obéit pas à la volonté du personnage. L'auteur ne semble indiquer les différentes stations du parcours que pour souligner l'absence de direction générale, l'ensemble de l'aventure étant ainsi marquée du sceau du hasard. Le héros de Dujardin tourne littéralement en rond. De ce point de vue, le chapitre VIII ${ }^{6}$, qui retrace la sortie inopinée et abrégée en fiacre, de la rue Stévens au parc Monceau et retour, est le symbole du cheminement du héros : c'est un cercle qui figure son rapport à l'espace, mais un cercle qui n'en est un qu'en raison du caprice de sa fiancée ; le déplacement s'annule par hasard et aurait dû conduire quelque part.

Le cas de Gustl est en apparence semblable. Lui aussi décrit un mouvement circulaire. Mais ce cercle dont il n'a pas lui-même conscience, est, contrairement à celui effectué par Prince dans le chapitre VIII des Lauriers sont coupés, hautement signifiant et, surtout, il recouvre le récit entier, lequel s'organise autour de cette structure spatiale circulaire. Ce parcours a souvent été commenté, si bien qu'il n'est pas nécessaire de s'y attarder ici trop longuement ${ }^{7}$. On en rappellera seulement les points saillants. Le chemin pris par le lieutenant au désespoir le conduit ainsi du centre-ville moderne - la salle de concert du Musikverein - au Prater, lieu extérieur par excellence, celui des plaisirs estivaux, lieu mixte entre la ville et la campagne, la civilisation impériale raffinée et assurée, d'une part, et la non-civilisation, presque sauvage, surtout la nuit, d'autre part. C'est là qu'il s'endort, sous les étoiles, seul et «nu » avant de retourner, le lendemain matin, vers la 105.

6. - Édouard Dujardin, Les Lauriers sont coupés, Paris, Flammarion, 2001, p. 96-

7. - $C f$. en particulier la présentation qu'en fait Konstanze Fliedl : Schnitzler, Stuttgart, Reclam, 2005, p. 119-120. 
culture qu'il a délaissée, vers ses repères symboliques de l'autorité et de l'État et du régime impérial dont il est lui-même l'expression et qui constituent et réassurent son identité - la cathédrale et la Résidence impériale ${ }^{8}$. Parti la veille du centre-ville dans un état psychologique désastreux, il remonte la pente dès qu'il repasse la barrière hautement signifiante du «Ring », et retrouve un univers familier. À l'échelle de la nouvelle entière, le chemin pris par Gustl au cours de cette nuit de crise identitaire est donc une route symbolique dont il faut décrypter la signification. On peut alors affirmer que, certes, le niveau de conscience de chacun des deux personnages est le même pour ce qui est de leur rapport à l'espace : tous deux ont l'impression d'errer dans la ville au gré du hasard. Mais la portée de ce déplacement n'est pas la même, puisque dans le cas des Lauriers sont coupés la figure de l'errance sans but est renforcée par l'organisation du récit alors que dans Leutnant Gustl le récit vise à donner à ce cheminement apparemment décousu un sens profond.

En ce qui concerne le rapport au temps, qui est l'autre élément déterminant dans un récit bref, les différences sont encore plus nettes et ont sur le plan narratif des conséquences plus essentielles. En apparence là aussi, l'impression prédomine d'une identité dans le traitement du temps, c'est même cela qui contribue parfois à accréditer la thèse d'une parenté entre les deux œuvres. Pourtant, et comme dans le traitement de l'espace, on doit nécessairement conclure à une opposition radicale. Il est vrai que Leutnant Gustl reprend une caractéristique fondamentale des Lauriers sont coupés : la scansion régulière du temps qui passe, aspect narratif d'autant plus frappant que dans les deux cas le temps de l'histoire est quasi identique - une soirée, quelques heures, une nuit ${ }^{9}$. En outre, le rapport entre temps du récit et temps de l'histoire, c'est-àdire entre la durée affichée de l'intrigue et celle, toute théorique, de la lecture du récit qui en est fait, est là aussi comparable ${ }^{10}$. Pourtant, le rapport des deux personnages au temps et à la durée n'est pas le même.

8. - Arthur Schnitzler, Leutnant Gustl und andere Erzählungen, Frankfurt a. M., Fischer TB (14941), 2001, p. 36-37.

9. - On ne saurait parler ici véritablement d'une différence entre les deux récits.

10. - Bien entendu, il s'agit là d'une approximation, dont l'étalon est tout subjectif. En fait, le rapport entre ces deux durées théoriques n'est pas le même, car Les Lauriers sont coupés est un récit quasiment deux fois plus long que Leutnant Gustl. Mais on pourra arguer que la nuit de Gustl, passée sous silence, allonge considérablement la durée de l'histoire, sans influer sur celle du récit, ce qui « équilibre » la comparaison entre les deux œuvres. Quoi qu'il en soit, c'est l'impression, le ressenti du lecteur et l'intention narrative de l'auteur, qui comptent dans ce domaine : or dans les deux cas, il est évident que l'auteur a voulu figurer un temps relativement court, dramatique dans son étendue réduite à quelques heures, dans un récit dont la longueur permet «normalement » de dépeindre une histoire nettement plus longue. Il en résulte une impression forte de dilution temporelle à la lecture, et ce sentiment est identique dans les deux textes. 
Pour résumer sans doute un peu schématiquement les choses, on peut dire que Gustl ressent le temps comme vide, il s'ennuie - l'ouverture de la nouvelle est bien connu, qui met en scène l'ennui du personnage : il se demande l'heure qu'il peut être et veut tirer sa montre au concert ${ }^{11}$ - et perd progressivement tous ses repères habituels, ce qui est la figuration concrète d'une psychologie de crise ${ }^{12}$. De son côté, Prince ressent lui aussi parfaitement le temps qui passe et il est possible de relever des signes d'ennui, mais le personnage est surtout contrarié par les reports que lui impose Léa, et son principal rapport à la durée se caractérise par l'impatience de retrouver enfin sa fiancée, et de pouvoir passer avec elle la nuit, comme il se le figure, depuis le début du roman ${ }^{13}$. Dans un cas, la scansion du temps qui passe sert à marquer la perte d'orientation psychologique d'un être en pleine crise identitaire : Gustl retrouve symboliquement un rapport au temps plus clair à la fin de la nouvelle, lorsqu'il a repris pied dans la société et qu'il prend conscience que tout danger de scandale est écarté. De l'autre, Prince ne perd à aucun moment la notion du temps, toute sa soirée - à l'exception du moment où il sombre dans le sommeil - est même placée sous le signe d'une réflexion sur la chronologie de ses derniers mois : dans le chapitre $\mathrm{V}$, il est chez lui et relit sa correspondance avec Léa, figurant ainsi au lecteur un rapport au temps sans ambiguïté ${ }^{4}$.

Après avoir rapidement fait le point sur le rapport au temps et à l'espace tel qu'il est présenté dans les deux textes, on s'aperçoit que l'ensemble des différences thématiques ou même formelles entre Leutnant Gustl et Les Lauriers sont coupés sont en réalité plus profondes qu'il n'y paraissait et que tout lien de ressemblance devient par là même problématique. En fait, les deux récits présentent des caractéristiques narratives opposées, rendant la comparaison finalement peu pertinente, même si elle est très tentante. En réalité, nous sommes bien face à deux projets narratifs et esthétiques tout à fait différents.

Une différence fondamentale entre les deux récits est évidente lorsqu'on adopte comme angle de lecture la notion de genre littéraire. Il semble qu'une telle lecture n'ait jamais été prise en considération dans le cas d'une étude de parenté entre Leutnant Gustl et Les Lauriers sont coupés. Sans accorder ici aux dénominations génériques plus d'importance qu'elles ne peuvent en avoir, force est cependant de constater que

11. - Leutnant Gustl, op. cit., p. 9.

12. - Konstanze Fliedl souligne le décalage de plus en plus net entre le marquage temporel extérieur et le sentiment de plus en plus problématique de la durée ressentie par Gustl : op. cit., (note 7), p. 119.

13. - La fin du chapitre VIII est éclairante : Les Lauriers sont coupés (cf. note 6) p. 101 ; Prince est ravi de la décision de Léa de mettre un terme prématurément à la sortie en fiacre.

14. - Ibid., p. 70-81. 
conformément à l'usage en vigueur à la fin du XIX ${ }^{\mathrm{e}}$ siècle en France, Dujardin n'a pas d'autre ambition dans ce domaine que d'écrire un « roman ». Dénomination vague s'il en est, qui ne repose sur aucun critère précis et ne sert ici à dénommer qu'un texte narratif en prose, dont la longueur n'est pas particulièrement remarquable. On le sait, la différence en France entre une «nouvelle » et un «roman » est floue et se limite à un vague critère de longueur très subjectif et fluctuant. Or dans la tradition germanique, le terme de « Novelle », très technique et riche de sens, correspond à des usages, discutables et parfois contradictoires, mais témoignant d'une réelle discussion théorique à partir du milieu du XIX ${ }^{\mathrm{e}}$ siècle. Le terme désigne un genre narratif qui n'est pas nécessairement très bref, mais qui propose au lecteur une structure narrative avérée et précise. Or c'est bien ce que fait Schnitzler, à plusieurs reprises, par le sous-titre qu'il choisit et dans sa correspondance ${ }^{15}$. Et il n'est pas difficile de retrouver dans ce texte des caractéristiques narratives évidentes du genre nouvellistique de langue allemande, tel qu'il s'est constitué dans la seconde moitié du siècle. Sans revenir dans le détail sur une question qui dépasserait le cadre de la présente étude, il convient de rappeler que le genre de la « Novelle », reprenant en cela en partie une définition goethéenne bien connue ${ }^{16}$, se présente comme un récit fortement structuré centré autour d'un événement de l'intrigue qui est constitué par l'auteur comme un point nodal, qu'il s'agisse d'un tournant narratif à proprement parler ou pas. Cette double caractéristique est bien présente dans Leutnant Gustl. En effet, et contrairement en cela aux Lauriers sont coupés, la nouvelle de Schnitzler repose bien sur un " événement inouï », c'est-à-dire, car c'est bien cela l'essentiel, un événement ressenti et présenté comme un facteur de désordre, comme l'irruption d'un élément perturbateur et générateur de chaos au sein d'un univers présenté au départ comme parfaitement en ordre. L'altercation entre Gustl et le " gros homme » ${ }^{17}$ remplit ce rôle à l'évidence, car l'insulte ressentie par le sous-lieutenant remet radicalement et immédiatement en cause tout ce qui assure la cohérence culturelle du monde dans lequel il vit. Tout dépend, au niveau de l'organisation de l'intrigue comme de celle du récit lui-même - la déambulation nocturne

15. - $C f$. les passages déjà cités, ainsi que d'autres témoignages de la genèse de Leutnant Gustl, cités par K. Fliedl (cf. note 7). Il parle, en accord avec un usage courant, de « Geschichte », mais désigne l'intrigue et non le texte lui-même, c'est-à-dire l'œuvre narrative dont il n'a jamais parlé autrement que comme d'une « Novelle».

16. - «Was ist eine Novelle anders als eine sich ereignete Begebenheit?» [« [...] Car qu'est-ce qu'une nouvelle, sinon un événement inouï qui s'est réellement passé. »], Johann Peter Eckermann, Gespräche mit Goethe in den letzten Jahren seines Lebens, Gespräch vom 29. Januar 1827, in: Artemis Gedenk-Ausgabe Bd. 24, hg. von. E. Beutler, Zürich, 1948, p. 224-225.

17. - Leutnant Gustl (cf. note 8), p. 16. 
- de ce coup de tonnerre initial ou quasi liminaire : en effet, Schnitzler prend soin, auparavant, de mettre en place un univers en ordre, avec ses codes, ses valeurs et son cadre hiérarchisé. Rien de tel dans le récit de Dujardin, qui n'est pas organisé autour d'un événement unique et essentiel. En outre, la « Novelle» se présente souvent sous l'apparence d'un récit circulaire, au sens où le désordre provoqué par l'événement a pris fin ou prend fin à l'issue de l'histoire. C'est d'ailleurs ce point qui distingue le genre classique des autres genres narratifs brefs plus modernes comme la Kurzgeschichte ou la nouvelle française, qui ne mettent pas systématiquement en scène ce phénomène de clôture. Une telle clôture narrative est évidente dans la nouvelle de Schnitzler : il s'agit de ce retour progressif à la situation de départ, que nous avons évoqué plus haut. Gustl retrouve ses repères, reprend le chemin du centre-ville et retourne à son quotidien. D'un point de vue psychologique, le personnage a sans doute changé, mais l'univers qui était le sien, lui, est identique ; toute l'ironie, toute l'amertume de l'auteur se révélant dans ce constat que le monde viennois de cette fin de siècle est immuable, incapable de changer, alors que les codes qui sont les siens ne sont plus d'actualité. Le salut de Gustl ne repose, on le sait, que sur la mort «providentielle », à tous les sens du terme, de son adversaire. Dans le récit de Dujardin, il serait sans doute abusif de parler d'absence de clôture ; cependant, on note la volonté de l'auteur de ne pas mettre en scène de pointe finale, ni même de structure tripartite typique de ce que les auteurs allemands ont conscience de faire en écrivant une « Novelle ». Les Lauriers sont coupés est un roman, bref, mais cela n'a ici pas d'importance. Et comme tout roman, ou même comme la plupart de ce que les Français nomment «nouvelle », la structure narrative n'est pas codifiée, et n'occupe pas, dans le projet esthétique de l'auteur, une place prépondérante. L'horizon d'attente du lecteur est tout autre. Le récit de Dujardin ne se présente absolument pas comme une « Novelle », sa structure - qui est bien réelle, cependant - ne repose pas sur le schéma ordre/désordre/retour à l'ordre, et ce pour la raison évidente que Dujardin, loin de centrer son texte sur un événement déterminant - central ou liminaire, comme dans Gustl -, a, au contraire, délibérément choisi de mettre en scène un vide événementiel. Et telle est bien la teneur de son projet ${ }^{18}$ : raconter la nuit d'un dandy parisien, selon le mode narratif particulier du monologue intérieur ; or cette existence se caractérise par un vide et une routine désespérants. Au début du chapitre V, Prince résume son parcours physique mais aussi son état d'esprit récurrent en utilisant le terme de « divagation ${ }^{19}$ ». Au-delà de

18. - $C f$. sur ce point les réflexions de Jean-Pierre Bertrand, dans son édition du texte $(c f$. note 6$)$, p. 18.

19. - Les Lauriers sont coupés (cf. note 6), p. 69. 
l'histoire vécue par le personnage, le récit lui-même peut s'apparenter à une divagation, c'est-à-dire à un cheminement linéaire, sans but, mais surtout sans ordre. Les lauriers sont coupés ne peut être comparé, en ce sens, à une « Novelle » de tradition germanique sans que l'on risque de méconnaître les intentions esthétiques de son auteur.

La question de la parenté entre les deux récits qui nous occupent devient dès lors problématique. Parler d'influence est possible, mais l'idée même d'un dialogue intertextuel conscient entre les deux auteurs doit être présentée avec circonspection. Deux exemples, deux cas particuliers permettent de mettre en lumière les différences essentielles entre les deux textes. Le premier est un motif commun aux deux histoires et il est systématiquement mis en avant : il s'agit de l'épisode du sommeil. Prince et Gustl s'endorment à un moment de l'histoire. Cependant, le rôle dévolu à ce motif dans le récit est très différent. Comme on le souligne avec justesse souvent, le sommeil de Gustl est le tournant narratif du récit, ce qui accentue d'ailleurs la parenté de la nouvelle de Schnitzler avec le genre allemand. À son réveil dans le Prater, l'officier reprend son chemin et rentre dans le centre-ville. Notons d'ailleurs que ce tournant narratif se produit juste après le mitan du récit ${ }^{20}$. Dans les Lauriers sont coupés, non seulement le sommeil de Prince est décrit ou plutôt mis en scène ${ }^{21}$, mais l'épisode se situe quasiment à la fin de l'œuvre 22 , et il ne s'agit pas d'un revirement pour le personnage, pas plus qu'on puisse y voir un tournant du récit. Le rêve de Prince est certes important, révélateur d'aspects essentiels, mais son rôle n'est en rien comparable à celui de Gustl. Le second exemple qui permet de mettre en évidence le fait que Les lauriers sont coupés et Leutnant Gustl sont l'expression de deux projets esthétiques radicalement différents est plus essentiel et concerne les descriptions et les jeux de regards. Étant donné la technique narrative choisie par Dujardin et Schnitzler, la difficulté majeure qu'ils devaient surmonter provient de ce que le lecteur n'a d'autre source d'information que ce que dit ou pense le personnage vecteur du récit. Or les deux auteurs apportent des solutions différentes à ce problème technique. Là où Schnitzler adopte, comme le font fréquemment les auteurs de « Novellen ${ }^{23}$ », un style

20. - Dans l'édition utilisée ( $c f$. note 8), la nouvelle s'étend de la p. 9 à la p. 42. Or l'ellipse consacrée au sommeil se situe p. 31 .

21. - K. Fliedl revient sur ce point et souligne avec pertinence le fait que c'est justement à ce moment que le monologue intérieur de Prince ressemble le plus à celui de Gustl, dans les formulations et la part faite à l'expression du sub-conscient. $C f$. note 7, p. 39-40.

22. - Dans le chapitre VII, p. 92 (le texte des Lauriers sont coupés s'étend de la p. 39 à la p. 115).

23. - Rappelons que le caractère dramatique de la nouvelle germanique a toujours été souligné, entre autres par Theodor Storm, qui a défini la « Novelle » comme sœur du 
très dramatique, Dujardin insiste plus souvent sur l'aspect purement descriptif. Schnitzler renonce à décrire le monde dans lequel évolue Gustl, laissant deviner l'apparence des choses et des lieux : on assiste à une réelle imitation des pensées du personnage, lequel n'a évidemment pas besoin de décrire ce qu'il voit. Dans le monologue du personnage de Dujardin, au contraire, on note de fréquentes remarques purement descriptives, qui sont, d'un point de vue technique, autant d'entorses au principe du monologue intérieur, et l'effet en est artificiel, voire maladroit ${ }^{24}$. Conséquence essentielle et remarquable, on s'aperçoit que dans Les lauriers sont coupés, le personnage vecteur de la narration est ainsi en permanence présenté en train de regarder et, surtout, de dire qu'il regarde ; cela peut sembler artificiel, mais il s'agit en réalité de l'essence même du projet de Dujardin. Son récit est une sorte d' « épiphanie du sujet », de « surgissement du 'je' 25 ». La mise en scène de cette subjectivité triomphante, affirmée et assurée, est aux antipodes de ce que Schnitzler prétend faire dans Leutnant Gustl. Loin de mettre en scène la victoire du moi sur le monde, on peut dire qu'il en présente en quelque sorte la défaite : Gustl est englouti par le monde qui l'entoure, ses codes et ses convenances, dont il ne peut se défaire et qui le mèneraient au suicide, si un deus ex machina ironique ne le sauvait in extremis. Et comme l'a montré le rapport au temps et à l'espace dans ce récit, tout concourt à y montrer la perte du moi, la disparition de la subjectivité. En d'autres termes, Schnitzler met en récit la primauté du monde sur le sujet. Et cela s'exprime par le biais du monologue intérieur qui contribue à montrer un personnage aux abois, tourné vers lui-même et pas vers la description du monde extérieur ; en outre, et au contraire, le lieutenant de Schnitzler se sent observé, scruté par le monde qui l'entoure. C'est un motif insistant, et quasiment absent chez Dujardin. Dès la scène d'ouverture de la nouvelle ${ }^{26}$, dont on peut se demander si la fonction première n'est justement pas de mettre en place ce jeu de regards permanents portés sur le personnage. Et il n'est pas jusqu'à l'incident central - l'altercation - qui ne soit présentée avant tout comme un jeu de regards : ceux des deux personnages, mais surtout celui que l'intrus porte sur Gustl, ceux que les témoins pourraient jeter

drame : «Schwester des Dramas ». $C f$. Theodor Storm, „Eine zurückgezogene Vorrede aus dem Jahre 1881“, in: Werke, Gesamtausgabe, hg. v. H. Engelhard, Bd. 3, Stuttgart, 1958, p. 524.

24. - Les Lauriers sont coupés (cf. note 6), p. 89 : « elle lève les bras au-dessus de la tête » ou encore : « je vais vers elle, auprès d'elle je m'assieds. »

25. - Expression de J.-B. Bertrand, (cf. note 6), p. 19-20.

26. - Au début, le moment est symbolique et déterminant, où Gustl prend conscience qu'une demoiselle, dans les loges, le fixe longuement. Leutnant Gustl, $c f$. note 8, p. 9 . 
sur la scène ${ }^{27}$. C'est donc bien la société viennoise qui est l'objet premier du récit de Schnitzler, au moins tout autant que la personnalité de Gustl. D'ailleurs l'auteur ne décrit pas son projet autrement :

Drei Dutzend Seiten genügen, um ein erstaunlich vollständiges Bild der österreichischen Republik zu entwerfen... ${ }^{28}$

Rien de tel n'est perceptible dans le roman de Dujardin. Certes, un certain univers parisien y est bien décrit. Mais l'objet du récit est bien plutôt la description d'un moi omniprésent, qui se présente sous l'apparence d'un regard porté sur tout et tous.

Au terme de cette brève étude, on ne peut que conclure à l'importance des différences qui existent entre les deux projets. Bien entendu, de telles différences sont pour certaines d'entre elles évidentes. Cependant, notre but est plutôt de souligner qu'il ne s'agit pas là uniquement de deux traitements différents d'une même thématique : Schnitzler ne s'est pas inspiré de Dujardin autrement que par la reprise d'une certaine technique narrative ; et encore faut-il sur ce point aussi apporter des nuances significatives, qui contribuent finalement à accréditer l'idée que la comparaison entre Les Lauriers sont coupés et Leutnant Gustl n'est pas entièrement légitime. On ne saurait rendre justice au texte de Schnitzler en le réduisant à être second dans l'expérimentation d'une technique narrative. En outre, on l'aura compris, il convient à notre sens de relever que ces deux textes sont chacun l'expression caractéristique d'une culture littéraire et narrative propre. Dujardin écrit un roman psychologique dont le projet doit être replacé dans le contexte de la Fin de siècle parisienne, friande d'une certaine écriture du moi finalement classique d'un point de vue technique, la modernité étant plutôt à chercher du côté du naturalisme ; Schnitzler quant à lui propose à son lecteur un texte éminemment viennois, dans sa conception comme dans sa réalisation : Leutnant Gustl est un exemple formel accompli d'une « Novelle » dont la structure se veut classique et de tradition germanique ; la modernité du projet réside dans l'expérimentation d'une nouvelle écriture de la psychologie.

27. - Gustl ne voit rien, quant à lui, il ne se rend pas immédiatement compte de ce qui retient sa main et son sabre. Ce n'est que dans un second mouvement qu'il voit la main de l'autre retenant la sienne.

28. - Arthur Schnitzler, mit Selbstzeugnissen u. Bilddokumenten dargestellt v. Hartmut Scheible, Reinbek b. Hamburg 1986, p. 79. 